Document downloaded from:

http://hdl.handle.net/10251/167122

This paper must be cited as:

Iborra, A.; Rodríguez-Alvarez, MJ.; Soriano, A.; Sánchez, F.; Bellido, P.; Conde, P.; Crespo, E... (2013). Effect of noise in CT image reconstruction using QR- Decomposition algorithm. IEEE. 5-9. http://hdl.handle.net/10251/167122

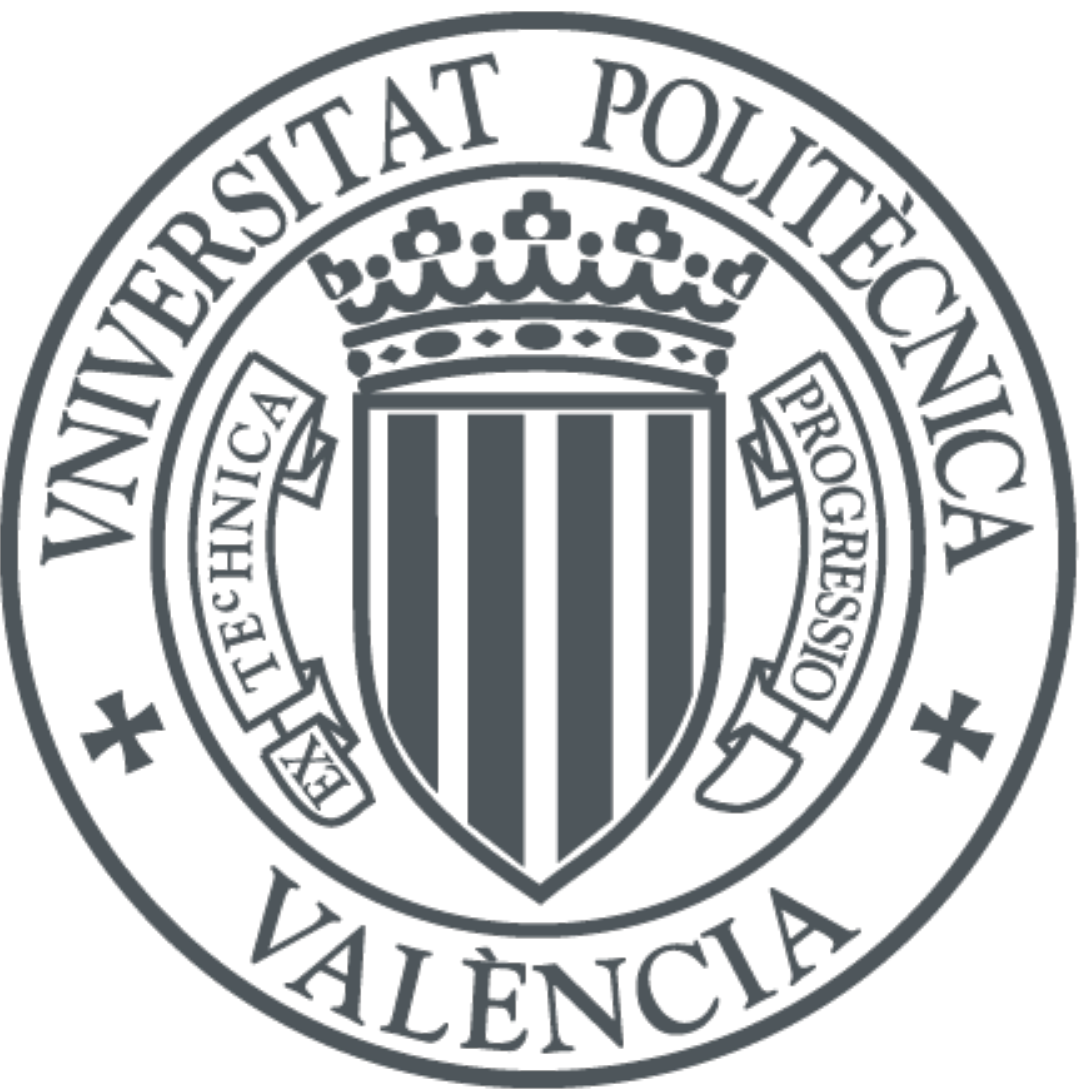

The final publication is available at

https://ieeexplore.ieee.org/xpl/conhome/6819718/proceeding

Copyright IEEE

Additional Information 


\title{
Effect of noise in CT image reconstruction using QR-Decomposition algorithm
}

\author{
A. Iborra*, M. J. Rodríguez-Álvarez, A. Soriano, F. Sánchez, P. Bellido, P. Conde, E. Crespo, A. J. González, \\ F. Martos, L. Moliner, J. P. Rigla, M. Seimetz, L. F. Vidal and J. M. Benlloch
}

\begin{abstract}
The QR-Decomposition algorithm for CT 3D image reconstruction uses a linear system of equations to model the CT system response. Linear systems have a condition number that can be used to estimate the image noise. In this work the number of projections and the number of pixels in the detector have been studied to characterize the $\mathrm{CT}$ and the linear system of equations. The condition number of the system is estimated for the previous parameters used to generate the CT model with the aim of characterizing how these parameters affect the condition number and therefore bound the image noise level. It is shown that the condition number mainly depends on the size of pixels of the detector rather than the number of projections and this algorithm can be applied to low dose CT 3D image reconstruction without compromising image quality.
\end{abstract}

Index Terms-Medical imaging, image reconstruction, QR decomposition, image noise

\section{INTRODUCTION}

$\mathbf{I}^{\mathrm{N}}$ $\mathrm{N}$ the field of CT 3D image reconstruction the Filtered Backprojection (FBP) [1] is the predominant algorithm nowadays, although recently, new alternatives are appearing such as MLEM [2] applied to CT 3D image reconstruction [3], [4], [5]. Another alternative for CT 3D image reconstruction is the resolution of a linear system of equations, $A x=b$, using QR-Decomposition with Givens rotations [6], where $A \in \mathbb{R}^{m \times n}$ is a matrix that models the CT system response in $3 \mathrm{D}$ (geometry and the conebeam factor [7]), $b \in \mathbb{R}^{m}$ is the CT measurement and $x \in \mathbb{R}^{n}$ represents the unknown scanned object.

The main advantage of this reconstruction method is that most of the computational effort lies in the decomposition process of $A$ in $Q$ and $R$. This process needs to be done only once, when the CT model is stated. The actual image reconstruction process only implies a matrix vector multiplication and a backward substitution process which have lower computational costs. The FBP has a computational cost of $O\left(N^{3}\right)$ and a matrix vector multiplication and a backward substitution process have a total computational cost of $O\left(2 N^{2}\right)$ which is one order of magnitude lower.

\section{Methods}

\section{A. Sensitivity of a linear system}

The matrix $A$ is a model and therefore an approximation. Likewise, the problem to solve is in fact to find $\hat{x}$ such that minimizes the residual $r$

$$
\min \|r\|_{2}=\min \|A \hat{x}-\hat{b}\|_{2}
$$

where $\hat{x}=x+\delta x$ and $\hat{b}=b+\delta b . \delta b$ represents the differences between the prediction of the model in $A$ of the CT measurement $b$ and the actual CT measurement $\hat{b}$. $\delta x$ represents the error in the image reconstruction, which is the difference between unknown scanned object $x$ and the object obtained by the reconstruction process $\hat{x}$.

It is expected that $\hat{x}$ and $x$ will be similar. In other words, if $\delta b$ is small, then $\delta x$ will be also small . In relative terms, it is expected that $\frac{\|\delta b\|_{2}}{\|b\|_{2}}$ and $\frac{\|\delta x\|_{2}}{\|x\|_{2}}$ will approximately have the same size. This is known as system sensitivity.

The condition number $(\kappa(A))$ of a matrix $A$ (described in detail in [8]) is a measurement of the sensitivity of the linear system $A x=b$

$$
\frac{\|\delta x\|_{2}}{\|x\|_{2}} \leq \kappa(A) \frac{\|\delta b\|_{2}}{\|b\|_{2}}
$$

If $A$ has a low condition number, then low values of $\frac{\|\delta b\|_{2}}{\|b\|_{2}}$ imply low values of $\frac{\|\delta x\|_{2}}{\|x\|_{2}}$ and it is said that the system is wellconditioned. If the condition number is high, then low values of $\frac{\|\delta b\|_{2}}{\|b\|_{2}}$ do not imply low values of $\frac{\|\delta x\|_{2}}{\|x\|_{2}}$ and it is said that the system is ill-conditioned. However, the difference between a well and an ill-conditioned system relies on the requirements of the problem that is being solved.

In this case, a linear system with a $\kappa(A) \approx 2$ will be considered as well-conditioned. The relative error of the reconstructed image of a linear system with this condition number will be, at most, twice the perturbation level introduced in $b$. However, in the case that the perturbation level introduced in $\mathrm{b}$ almost reaches the accuracy requirement of the problem a lower $\kappa(A)$ may be needed.

\section{B. QR-Decomposition algorithm overview}

The algorithm of 3D image reconstruction using QRDecomposition (described in detail in [9]) consists of the generation of a linear system of equations $A x=b$, where $A$ is the CT system response. Since the model is an approximation, the problem to solve becomes the problem (1), where a QRDecomposition is applied in $A$ using Givens rotations

$$
\min \|r\|_{2}=\min \|Q R \hat{x}-\hat{b}\|_{2}
$$




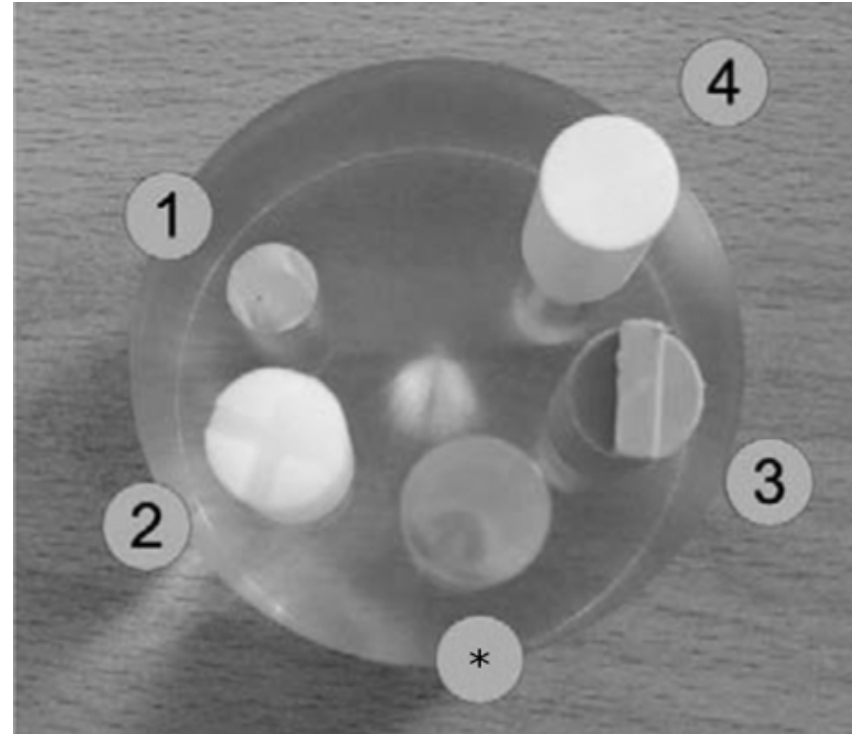

Fig. 1. Phantom of PMMA with inserts of PMMA (*) for alineation purposes, air (1), polytetrafluoroethylene (PTFE) (2), polyethylene (PE) (3) and polyoxymethylene (POM) (4), which model regions filled with air inside the body, soft bone, adipose tissue, and organs tissue, respectively.

where $Q \in \mathbb{R}^{m \times m}$ is orthogonal and $R \in \mathbb{R}^{n \times n}$ is upper triangular.

As $Q$ is orthogonal

$$
\begin{aligned}
& \min \|r\|_{2}=\min \left\|Q^{T} Q R \hat{x}-Q^{T} \hat{b}\right\|_{2} \\
& \min \|r\|_{2}=\min \left\|R \hat{x}-Q^{T} \hat{b}\right\|_{2}
\end{aligned}
$$

where $x$ (the reconstructed image) is found by means of a backward substitution process.

\section{Measurements}

CT measurements have been generated based on a real CT measurement from the Albira $\mu \mathrm{CT}$ [10] of a polymethylmethacrylate (PMMA) cylinder of $50 \mathrm{~mm}$ height and $55 \mathrm{~mm}$ in diameter containing smaller cylinders of different materials of $8 \mathrm{~mm}$ in diameter at $16 \mathrm{~mm}$ off the axis (see figure 1). Using this reconstruction as a geometrical guide, a virtual phantom is generated with the ideal CT value (depending on the material) in each cylinder zone (see figure 2).

The matrix $A$ (CT system response model) is generated according to the geometry of the Albira $\mu \mathrm{CT}$. Considering that the digital phantom is generated according to the geometry of an Albira $\mu \mathrm{CT}$ reconstruction, the generated measurements of the CT system response model and the measurements of the Albira $\mu \mathrm{CT}$ can be compared and the model can be verified.

In order to generate the measurements, the digital phantom, $x$, is multiplied by the CT system response model, $A$, to obtain the measurement expected by the model, $b$. Then a randomly generated $\delta b$ is added to $b$ such that $\frac{\|\delta b\|_{2}}{\|b\|_{2}}$ raises to a desired percentage $(1 \%, \ldots, 10 \%)$. Since $\delta b$ is randomly generated, to reduce statistical fluctuations, ten $\delta b$ are generated for each combination of number of projections and number of pixels in the detector.

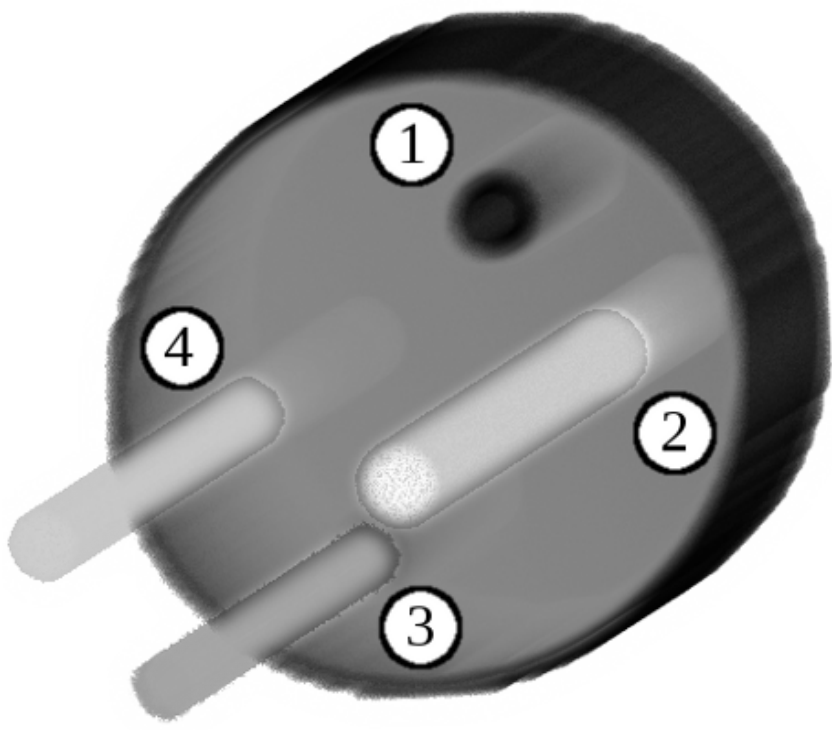

Fig. 2. Digital phantom of PMMA with inserts of air (1), PTFE (2), PE (3) and POM (4). Half of the PMMA cylinder has been removed in order to see the inserts.

\section{Condition number estimation}

The condition number of $A$ is estimated to find out how the parameters of the CT model influence on it. These parameters are: number of pixels in the CT detector, number of elements in the reconstructed image (voxels) and $\delta b$ (difference between the predicted measurement and the obtained measurement).

The CT model was generated varying the number of projections from 80 to 260 and the number of pixels in the detector from $60 \times 60$ to $192 \times 192$. For all generated CT system response models, the sizes of the detector panel and the reconstructed image are constant (and the same as in the Albira $\mu \mathrm{CT}$ ). Therefore, to model a higher number of pixels in the detector implies the reduction of the pixel size. In the same way, reconstructing an image with higher resolution implies that this image will have a higher number of elements. Each of these models is used to reconstruct images with perturbed measurements such that $\frac{\|\delta b\|_{2}}{\|b\|_{2}}$ varies from $1 \%$ to $10 \%$.

A 3D image reconstruction is performed for each $\hat{b}$ and its relative error, $\frac{\|x-\hat{x}\|_{2}}{\|x\|_{2}}$, is calculated. The condition number estimation relies on the relation between the average of the ten relative errors of a combination of model parameters and the perturbation level added to $b$ according to bound (2).

\section{RESULTS}

An estimation of the condition number of $A$ is obtained for each combination of number of projections and number of pixels in the detector. As it was expected, the estimated condition number decreases as the number of pixels in the detector and number of projections increase. However, the condition number decreases mainly with the number of pixels in the detector (see figures (3) and (4)).

For the sake of simplicity, the size of each pixel in the detector and the size of each image element is taken into 


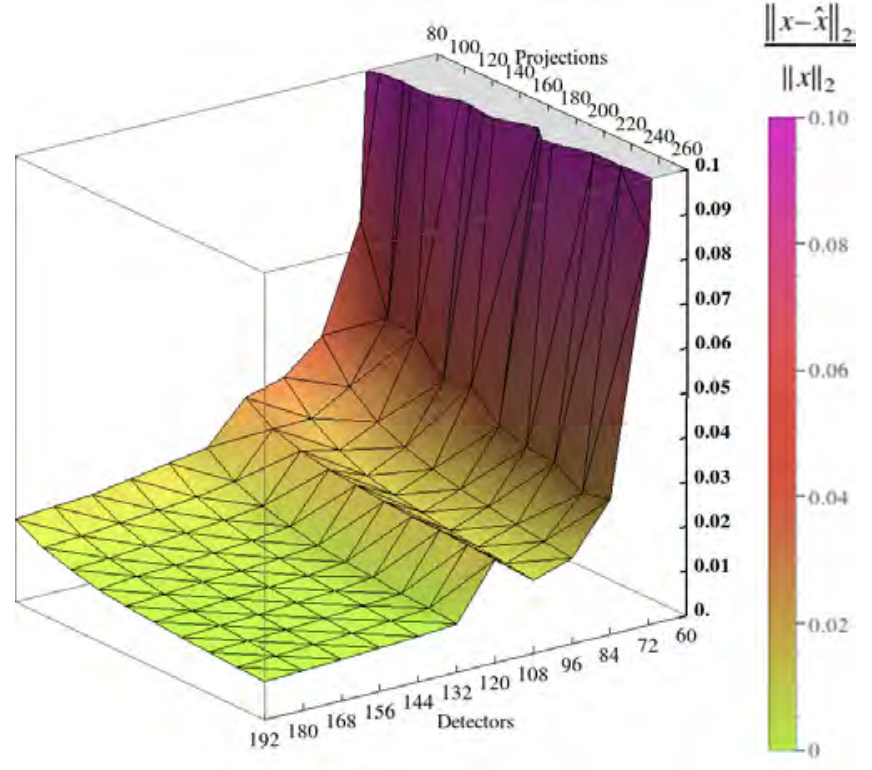

Fig. 3. Average relative errors of image reconstructions of all combinations of the CT system response model with $\frac{\|\delta b\|_{2}}{\|b\|_{2}}=1 \%$.

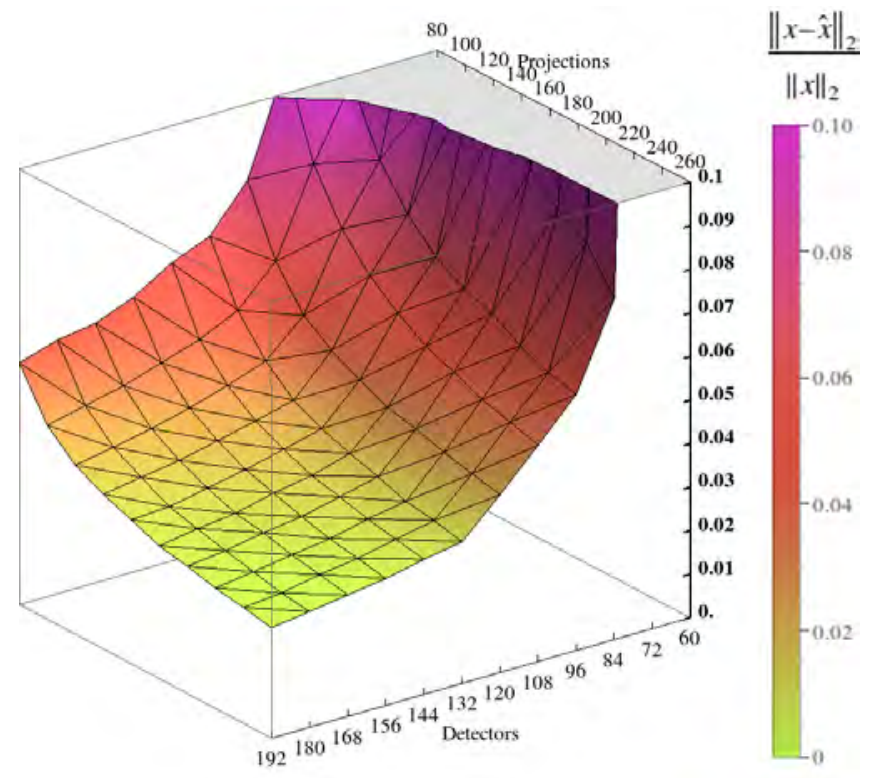

Fig. 4. Average relative errors of image reconstructions of all combinations of the CT system response model with $\frac{\|\delta b\|_{2}}{\|b\|_{2}}=3 \%$.

account by the ratio

$$
R=\frac{\text { voxel size }}{\text { detector size }}
$$

Obtained data show that for $R$ above $2.2 \mathrm{CT}$ system models have a $\kappa(A) \approx 2$ (the relative error of the reconstructed image is twice the perturbation level introduced in $b$ at most), even with a number of projections which is scarce for other reconstruction methods (as FBP). Similar results are obtained for various resolutions (see table I). Figures (5) - (9) show the mean relative error of reconstructions for different $R$ values

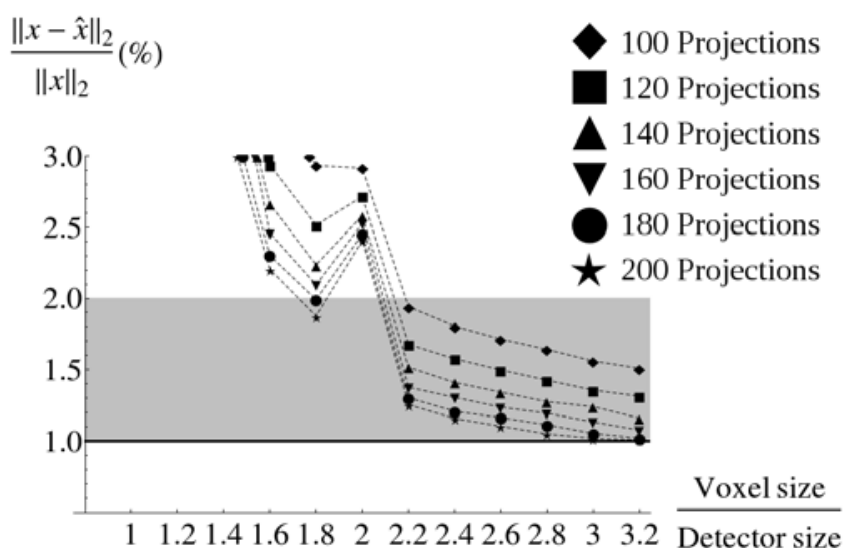

Fig. 5. Average relative errors of image reconstructions for different $R$ values with $1 \%$ level of $\mathrm{CT}$ measurement perturbation.

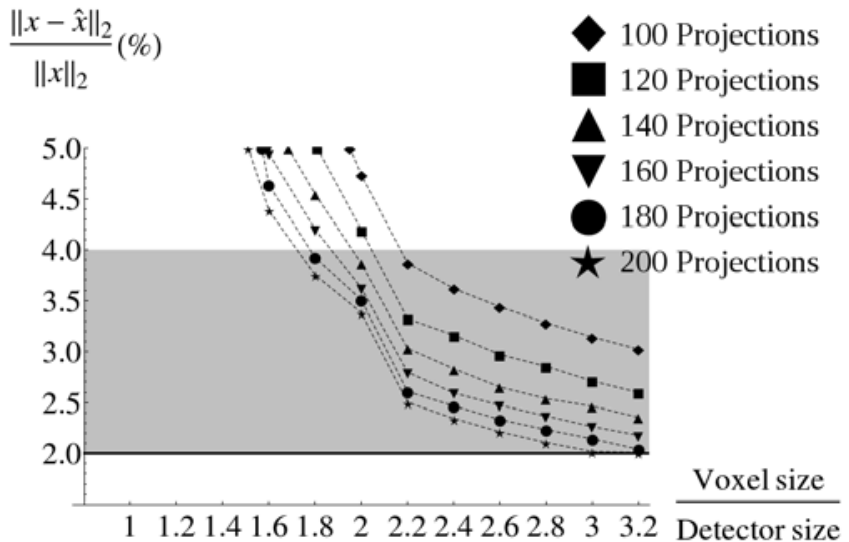

Fig. 6. Average relative errors of image reconstructions for different $R$ values with $2 \%$ level of $\mathrm{CT}$ measurement perturbation.

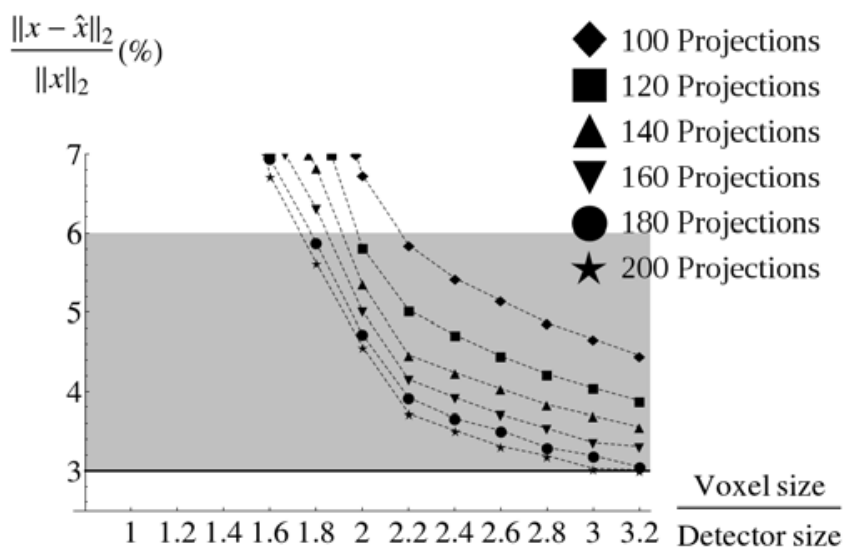

Fig. 7. Average relative errors of image reconstructions for different $R$ values with $3 \%$ level of CT measurement perturbation.

(only $1 \%$ to $5 \%$ are shown). A horizontal line shows the level of CT measurement perturbation in each case and shaded area represents the relative errors below the bound (2) derived from a $\kappa(A)=2$. 


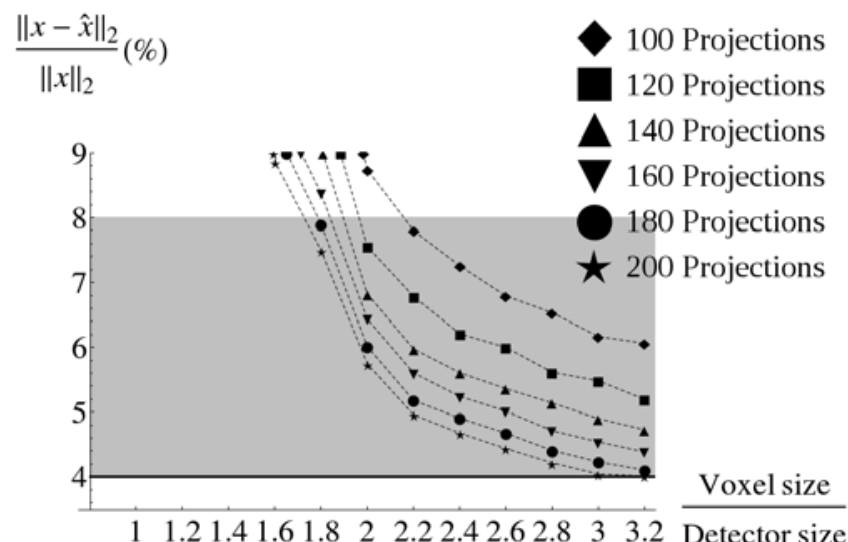

Fig. 8. Average relative errors of image reconstructions for different $R$ values with $4 \%$ level of CT measurement perturbation.

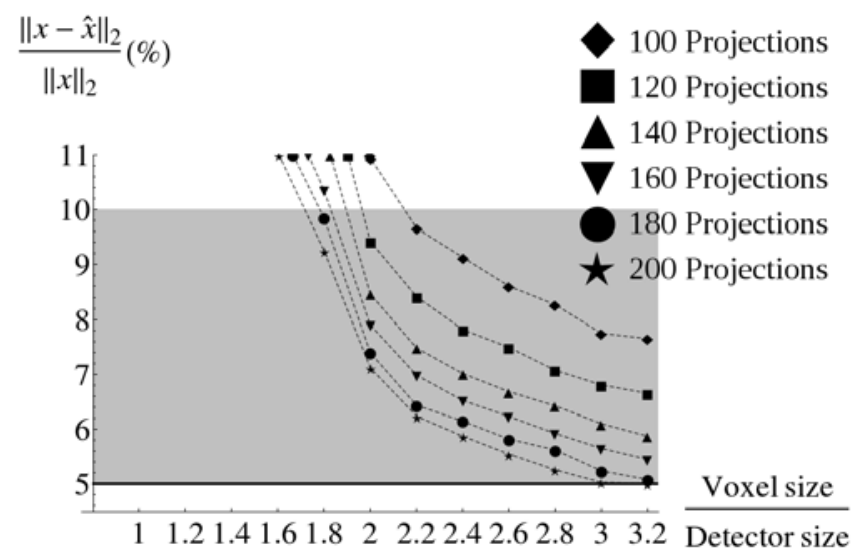

Fig. 9. Average relative errors of image reconstructions for different $R$ values with $5 \%$ level of CT measurement perturbation.

TABLE I

MEAN RELATIVE ERRORS FOR CONFIGURATIONS THAT MATCH THE PIXEL / VOXEL RATIO AND HAVE ONLY 100 PROJECTIONS AS THE REQUIRED RESOLUTION INCREASES.

\begin{tabular}{|l||c|c|c|}
\hline Voxel Size & $2.17 \mathrm{~mm}$ & $1.30 \mathrm{~mm}$ & $0.93 \mathrm{~mm}$ \\
\hline Detector / Voxel ratio & 2.33 & 2.20 & 2.28 \\
\hline Relative error at 1\% noise level & 0.0134 & 0.0196 & 0.0215 \\
\hline Relative error at 2\% noise level & 0.0288 & 0.0383 & 0.0436 \\
\hline Relative error at 3\% noise level & 0.0441 & 0.0574 & 0.0670 \\
\hline
\end{tabular}

\section{CONCLuSions}

The condition number of the model matrix of the QRDecomposition algorithm for CT 3D image reconstruction mainly depends on the ratio between the sizes of detectors and voxels. For values of $R>2.2$ the condition number of its model matrix let us bound the image noise up to a maximum of twice the difference between the predicted measurement and the CT measurement, even with a number of projections that for other algorithms, as FBP, is scarce.

The requirements for $\frac{\text { voxel size }}{\text { detector size }}>2.2$ for high resolution CT 3D image reconstruction in terms of number and size of the pixels of the CT detector are achieved or exceeded in modern
CT scanners. Therefore, the QR-Decomposition algorithm can be used in low dose CT imaging, where a limited number of projections are required without compromising image quality.

\section{REFERENCES}

[1] L. A. Feldkamp, L. C. Davis, and J. W. Kress, "Practical cone-beam algorithm," Journal of the Optical Society of America A, vol. 1, p. 612, June 1984.

[2] L. A. Shepp and Y. Vardi, "Maximum likelihood reconstruction for emission tomography.," IEEE transactions on medical imaging, vol. 1, pp. 113-22, Jan. 1982.

[3] K. Lange and R. Carson, "EM reconstruction algorithms for emission and transmission tomography.," Journal of computer assisted tomography, vol. 8, pp. 306-16, Apr. 1984.

[4] M. Beister, D. Kolditz, and W. A. Kalender, "Iterative reconstruction methods in X-ray CT.," Physica medica : PM : an international journal devoted to the applications of physics to medicine and biology : official journal of the Italian Association of Biomedical Physics (AIFB), vol. 28, pp. 94-108, Apr. 2012.

[5] A. Soriano, M. J. Rodríguez-Alvarez, A. Iborra, F. Sánchez, M. Carles, P. Conde, A. J. González, L. Hernández, L. Moliner, A. Orero, L. F. Vidal, and J. M. Benlloch, "EM tomographic image reconstruction using polar voxels," Journal of Instrumentation, vol. 8, pp. C01004-C01004, Jan. 2013.

[6] M.-J. Rodríguez-Álvarez, F. Sánchez, A. Soriano, and A. Iborra, "Sparse Givens resolution of large system of linear equations: Applications to image reconstruction," Mathematical and Computer Modelling, vol. 52, pp. 1258-1264, Oct. 2010.

[7] W. Yao and K. Leszczynski, "Analytically derived weighting factors for transmission tomography cone beam projections.", Physics in medicine and biology, vol. 54, pp. 513-33, Feb. 2009.

[8] D. S. Watkins, Fundamentals of Matrix Computations. John Wiley and Sons. Inc., 2002

[9] G. H. Golub and C. F. V. Loan, Matrix Computations. JHU Press, 1996.

[10] F. Sánchez, A. Orero, A. Soriano, C. Correcher, P. Conde, A. González, L. Hernández, L. Moliner, M. J. Rodríguez-Alvarez, L. F. Vidal, J. M. Benlloch, S. E. Chapman, and W. M. Leevy, "ALBIRA: a small animal PETSPECTCT imaging system.," Medical physics, vol. 40, p. 051906, May 2013. 